\title{
Determination of filtration and consolidation properties of protein crystal suspensions using analytical photocentrifuges with low volume samples
}

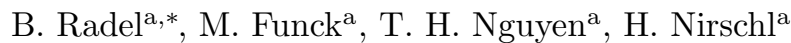 \\ ${ }^{a}$ Institute of Mechanical Process Engineering and Mechanics, Karlsruhe Institute of Technology, Strasse am Forum 8, 76131 Karlsruhe, \\ Germany
}

\begin{abstract}
Proteins and in particular pharmaceutical proteins like monoclonal antibodies are expensive products. Protein crystallization is an interesting alternative process step to the common purification and formulation of such proteins. The solid-liquid separation of proteins however, is a challenging task because the proteins are sensitive to mechanical stress, which could lead to crystal breakage and often are only available in small amounts for research and development. In this study a newly developed filtration cell for the LUMiSizer $\AA$ centrifuge is used to analyze the filtration behavior with low volume samples. Isometric and needle shaped lysozyme crystals serve as model protein crystals. The needle shaped crystals showed about twice as high compressibility and much higher cake resistance than the isometric lysozyme crystals. After the filtration at maximum pressure in the centrifuge the mean particle diameter decreased compared to the unstressed median diameter. With the filtration cell the cake height and filter cake compression can be monitored in situ and the filter cake resistance and solids volume fraction can be calculated with one experiment using 200 to $300 \mu l$ sample.
\end{abstract}

Keywords: Centrifugal filtration; Cake filtration; Protein crystals;

\section{Introduction}

In the pharmaceutical, food and biotechnology industries enzymes and other proteins are used widely (Walsh, 2002). Advances in the biotechnology industries have led to an efficient fermentation step which results in high product titers. Hence, the downstream processing of pharmaceutical proteins, where the purification consists of several chromatography steps, is an expensive process (Walsh, 2002; Kayser, 2005; Schmidt et al., 2005). A different and less expensive method of purifying proteins would be to implement a selective crystallization step in the downstream processing. Such a crystallization step as proposed by Schmidt et al. (2005); Hekmat et al. (2007) can serve as a purification but also as a formulation step. Crystallized proteins also offer additional advantages like for instance extended shelf life, easier product handling and different drug release properties (Jen and Merkle, 2001; Basu et al., 2004). After the proteins have been crystallized, they have to be separated from the mother liquor. Therefore, a solidliquid separation step is necessary.

Filtration is a widely used method in the pharmaceutical, chemical or food industries to separate a dispersed phase from a continuous phase using a porous filter medium (Alles and Anlauf, 2003). The filtration process itself has been well characterized by Tiller and Hsyung (1993); Tiller

\footnotetext{
*Corresponding author

Email address: benjamin.radel@kit.edu (B. Radel)
}

and Kwon (1998). To successfully optimize the filtration process, knowledge about the resistance and the consolidation properties is required. Those properties have to be experimentally determined for each material, particle shape and size distribution. Typically, dead-end filtration experiments with a compression-permeability device are used to determine the solids volume fraction and the filter cake resistance (Alles, 2000; Loginov et al., 2014). This method is well understood but has some limitations. The material behavior at low pressures is hard to characterize and the method is not suitable for low volume samples (Loginov et al., 2014).

Sambuichi et al. (1987) analyzed centrifugal batch filtration and found that due to the accelerated sedimentation in the centrifugal field, a clear supernatant forms which is followed by the turbid suspension. They developed an experimental setup for the filtration in the centrifugal field. The transparent filter basket rotated in a horizontal plane and the liquid and suspension position were measured with a camera using stroboscopic light to produce a still image. The centrifugal accelerations and thus the pressure were low in their approach due to difficulties to track the suspension interface at higher speeds. Recently Loginov et al. (2014, 2017) introduced a method to use centrifugal filtration with an analytical photocentrifuge described by Lerche and Sobisch (2007). In their approach the filtrate volume is measured in situ. The filter cake and the suspension however, cannot be monitored directly. 
In contrast to conventional organic or inorganic crystals the protein crystals are much more fragile. Cornehl et al. found that even low mechanical stress can lead to crystal breakage or crystal abrasion. The smaller fragments lead to a decrease of the filter cake porosity and thus making the solid liquid separation a challenging task (Cornehl et al., 2013, 2014). Therapeutic proteins, especially monoclonal antibodies are expensive products. According to Farid (2007) the costs of goods per gram for a production rate of $100 \mathrm{~kg}$ per year using Chinese Hamster ovary cells as expression system lies between 300 and 3000 USD. Hence, to analyze the filtration behavior and to design the solid-liquid separation process a method using small volume samples would be advantageous to reduce the costs.

In this work we present a newly designed filter cell which can be used to monitor the filtration process with an analytical photocentrifuge. With the cell the filter cake height, the solids volume fraction and the filtration resistance can be monitored or calculated directly from the very same experiment requiring only 200 to $300 \mu \mathrm{l}$ of sample. Isometric and needle shaped lysozyme crystals were used as a model system in this study.

\section{Theory}

\subsection{Protein crystallization}

\subsubsection{Lysozyme}

Lysozyme is an enzyme with antibacterial properties with a density of $1330 \mathrm{~kg} \mathrm{~m}^{-3}$. Due to its antibacterial effect it is often used in the food industries to increase the shelf life of cheese. Hen-egg white lysozyme is available on a large scale and has been well characterized (Durbin and Feher, 1986; Howard et al., 1988; Koizumi et al., 2004; Hekmat et al., 2007; Liu et al., 2010; Zamiri and De, 2010; Eichholz et al., 2011; Cornehl et al., 2013, 2014; Liu et al., 2016). Because many properties have already been investigated and its good and inexpensive availability, lysozyme is a good model protein for this study.

\subsubsection{Crystallization of lysozyme}

In a crystal the molecules are in a highly ordered structure. To obtain crystals from a liquid solution one has to create an supersaturation, meaning that the dissolved amount of protein is above the equilibrium concentration, which leads to a meta stable equilibrium. There are several methods to achieve this. One method would be by liquid evaporation, either through temperature increase or by using vacuum evaporation. Other methods decrease the solubility by adding precipitants. Most proteins denature at low or high temperatures, thus the better approach for biomolecules is either vacuum evaporation or precipitant addition. Usually the resulting crystal concentration using vacuum evaporation is much higher but the process is more energy demanding. On a small scale the precipitation of the protein is often used, but the scale-up of such a crystallization process is difficult, because a homogeneous distribution of the precipitant has to be achieved to ensure a narrow distribution of crystal properties.

Depending on the crystallization conditions, different crystal structures can be observed. In the case of lysozyme there are for instance isometric, rod-like and needle shaped crystals. Exemplary microscope images of those crystals can be seen in Fig. 1. The crystallization of lysozyme has already been described in literature. Howard et al. (1988); Hekmat et al. (2007); Aldabaibeh et al. (2009) investigated the solubility of lysozyme in a sodium acetate buffer at various $\mathrm{pH}$ values and with different concentrations of the precipitant sodium chloride. They found that an increase of sodium chloride concentration leads to a decrease of the lysozyme solubility. Hence, the sodium chloride serves as a precipitant. The bulk crystallization of lysozyme using vacuum evaporation was performed by Groß and Kind (2016); Barros Groß and Kind (2017). Isometric crystals were produced using no salt at room temperature. Using a high sodium chloride concentration needle shaped crystals could be generated.

\subsection{Mechanical stability of lysozyme}

The mechanical properties of single lysozyme crystals like Young's modulus and Vickers hardness have already been investigated (Morozov and Morozova, 1981; Tait et al., 2008; Zamiri and De, 2010). Tait et al. found the hardness to be $15 \mathrm{MPa}$ for moist lysozyme crystals. For dried crystals the hardness increases to $96 \mathrm{MPa}$ after two days of drying and reaches $200 \mathrm{MPa}$ after 10 days of drying. The Young's modulus also increases from 0.49 to $4.2 \mathrm{GPa}$ after drying. This indicates a very significant influence of moisture on the mechanical properties of the crystals. Koizumi et al. discovered a temperature dependence of the crystal hardness in the case of lysozyme. In their study the hardness dropped strongly from about $20 \mathrm{MPa}$ at $17^{\circ} \mathrm{C}$ to about $10 \mathrm{MPa}$ at $32{ }^{\circ} \mathrm{C}$ (Koizumi et al., 2004).

The compressive breakage behavior of lysozyme crystals was studied by Cornehl et al. (2014). With a compression-permeability cell and a pressure filter cell Cornehl et al. investigated the breakage for different pressures, the kinetics of the crystal breakage and also the changes in filter cake resistance and porosity. The crystal breakage happens quickly because after the first minute of compression further crushing of crystals cannot be observed. Also,
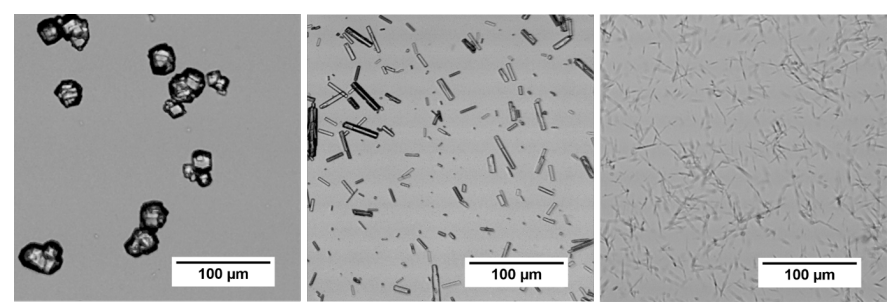

Figure 1: Microscope images of left: Isometric crystals; Middle: Rodlike crystals; Right: Needle shaped crystals of the model protein lysozyme 
crystal breakage occurs at very low pressures. Cornehl et al. mention that from $0.25 \times 10^{5}$ to $1.01 \times 10^{5} \mathrm{~Pa}$ the breakage progresses steadily but at pressures higher than $1.01 \times 10^{5} \mathrm{~Pa}$ the particle size does not change anymore. The porosity and the specific filter cake resistance are dependent on the pressure difference indicating compressible material behavior. Also, the crystal shape has a big influence on the porosity and resistance and thus on the filterability of the particle system.

\subsection{Filtration}

Filtration is a mechanical process of solid-liquid separation. A suspension is split into filtrate and retentate by interaction of disperse phase with a porous filter medium which retains the particles in or on the filter medium. The driving force is the pressure difference between the retentate and the filtrate. Pressure can be either applied on the retentate side (pressure filtration) or one can apply a vacuum at the filtrate side (vacuum filtration). In cake filtration the particles are retained at the surface of the filter medium and form particle bridges. The resulting filter cake also serves as a filter medium itself (Rushton et al., 2000).

\subsubsection{Cake filtration}

The filter cake and the filter medium lead to a pressure drop in the system because of flow resistance due to friction. For constant pressure filtration the Darcy equation:

$$
\frac{\mathrm{d} V}{\mathrm{~d} t}=\frac{\Delta p \cdot A_{m}}{\eta \cdot R}
$$

correlates the volume flow with the pressure drop across the filter cake $\Delta p$, the filter area $A_{m}$, the liquid's viscosity $\eta$ and the total resistance $R$. The total resistance $R$ can be split into the resistance of the filter cake $R_{c}$ and the resistance of the filter medium $R_{m}$ :

$$
R=R_{c}+R_{m}
$$

Since the cake resistance is strongly dependent on the amount of particles in the filter cake it is usually normalized with the filter cake height $h_{c}$ to make the resistances comparable. The normalized height specific cake resistance $\alpha_{c}$ and the specific cake permeability $K$ are defined as:

$$
\alpha_{c}=\frac{R_{c}}{h_{c}}=K^{-1}
$$

The filter cake can either be incompressible, meaning that the porosity $\epsilon$ and filter cake height are independent of the applied pressure or compressible which means that the porosity decreases and the filtration resistance increases with higher pressure. The porosity and the solids volume fraction are defined as

$$
\epsilon_{c}=\frac{V_{h}}{V_{c}}=\frac{V_{c}-V_{s}}{V_{c}}=1-\phi_{c}
$$

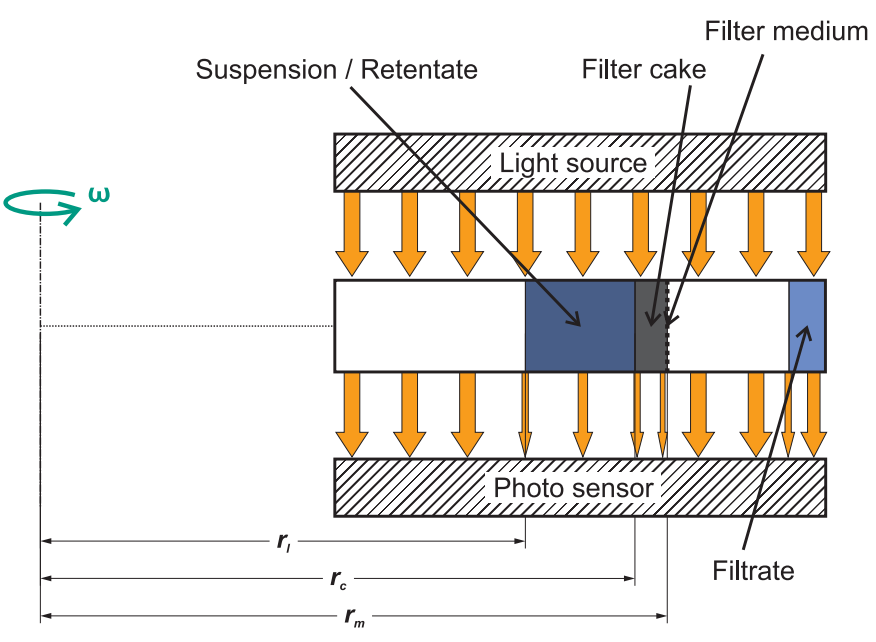

Figure 2: Schematic drawing of the setup for the centrifugal cake filtration

with $V_{h}$ as the total volume of the pores, $V_{s}$ as the volume of the solids and $V_{c}$ as the total volume of the filter cake. According to Alles and Anlauf (2003) an efficient filtration has a low specific filtration resistance (fast filtration) and a high solids volume fraction in the filter cake (less fluid in the filter cake). Tiller and Kwon (1998) proposed an empirical approach to describe the solids volume concentration and the cake resistance:

$$
\begin{aligned}
& \alpha=\alpha_{0} \cdot\left(1+\frac{p}{p_{0}}\right)^{N}, \\
& \phi=\phi_{0} \cdot\left(1+\frac{p}{p_{0}}\right)^{\beta} .
\end{aligned}
$$

In Eqs. 5 and 6 the parameters $N$ and $\beta$ have to be fitted to measurement data. The parameters $\alpha_{0}, \phi_{0}$, and $p_{0}$ are a measured reference point. Often the measured data point at the lowest pressure is used. According to Alles (2000); Alles and Anlauf (2003), the sum of the exponents $N$ and $\beta$ is an indicator for the compressibility of the system. If the sum is zero, the filter cake is incompressible. Values between 0 and 1 indicate a compressible system, numbers between 1 and 2 indicate high compressibility and values above 2 indicate super compressibility.

\subsubsection{Centrifugal cake filtration}

Filtration experiments are usually conducted at constant pressure or constant filtrate flow. To perform filtration experiments in the centrifuge a filtration cell must be used. The basic design of such a filtration cell is depicted in Fig. 2. In the centrifuge the pressure difference is generated by the centrifugal field. Hence, the pressure at the filter membrane decreases during the filtration, which means differential balance equations must be used. Previous studies (Barr and White, 2006a,b) developed a compression rheological model for the cake formation and draining using drum centrifugation. Loginov et al. (2014, 2017) used a similar approach as presented in this study. 
They used a filtration cell and monitored the filtrate flow by measuring the light transmission during the filtration with the analytical photocentrifuge LUMiSizer@) (LUM $\mathrm{GmbH}$, Germany) and derived formulas to calculate the filter resistance in the centrifugal field based on the Darcy's equation. This is achieved by tracking the time-resolved position of the gas-liquid interface in the filtrate chamber. Each phase interface depicted in Fig. 2 leads to a characteristic signal in the transmission profile. Hence, for each time step the radial position of the filtrate can be determined. If the cross-sectional area of the filtrate chamber is constant the filtrate flow can be easily calculated. In their approach however the filter cake and the liquid or suspension above the filter cake are not visible. The differential pressure in the centrifugal field can be calculated according to:

$$
\mathrm{d} p=\rho \cdot \omega^{2} \cdot r \cdot \mathrm{d} r .
$$

The total pressure at the filter membrane cannot be calculated directly, hence it has to be split up into the hydraulic pressure of the liquid above the filter cake $\left(\Delta p_{l}\right)$, the solids network pressure $\left(\Delta p_{s}\right)$ and the pore fluid pressure $\left(\Delta p_{\text {cap }}\right)$, which can be obtained by integration of Eq. 7. Crucial for the pressure calculation are the positions of the liquid interface $r_{l}$, the position of the filter cake $r_{c}$ and the position of the filter membrane $r_{m}$. With the setup depicted in Fig. 2 and the LUMiSizer@ the light transmission through the filtration cell allows to determine those positions.

$$
\begin{aligned}
\Delta p_{l} & =\frac{\rho_{l} \cdot \omega^{2}}{2} \cdot\left(r_{c}^{2}-r_{l}^{2}\right), \\
\Delta p_{s} & =\frac{\rho_{s} \cdot \phi \cdot \omega^{2}}{2} \cdot\left(r_{m}^{2}-r_{c}^{2}\right), \\
\Delta p_{c a p} & =\frac{\rho_{l} \cdot(1-\phi) \cdot \omega^{2} \cdot S}{2} \cdot\left(r_{m}^{2}-r_{c}^{2}\right) .
\end{aligned}
$$

If the solids and liquid densities $\left(\rho_{s}, \rho_{l}\right)$, the saturation and distribution of liquid in the filter cake $(S)$ are known, the total pressure can be calculated with the knowledge of the volume concentration $(\phi)$.

Since the initial volume concentration $\left(\phi_{0}\right)$ is known and the cross-sectional area in the cuvette is constant, the solids volume fraction can be calculated according to:

$$
\phi=\phi_{0} \cdot \frac{h_{s, 0}}{h_{c}} .
$$

The height of the filter cake $\left(h_{c}\right)$ and the initial height of the suspension $\left(h_{s, 0}\right)$ can be directly obtained from the transmission profiles of the LUMiSizer@. If the filter cake is permeated with clear liquid the position of the filter cake $r_{c}$ is visible and the cake height equals:

$$
h_{c}=r_{m}-r_{c} \text {. }
$$

For a filtration experiment the particles settle during the filtration and according to Sambuichi et al. (1987) a clear zone with supernatant, a turbid zone with particles and the filter cake itself form. In this case the filter cake position is only visible at the very end of the experiment. That is why the height of the filter cake is calculated according to:

$$
h_{c}=\frac{\left(r_{l, 0}-r_{s}\right) \cdot \phi_{0}}{\phi_{c}}
$$

with $r_{s}$ as the position of the sedimentation front. With the knowledge of the fluid and particle densities, the liquid's viscosity, the positions of the filter cake, the height of liquid above the filter cake and the initial liquid height, the filter resistance can be calculated with the Darcy equation as shown previously by Loginov et al. (2014):

$$
\frac{\mathrm{d} h}{\mathrm{~d} t}=\frac{1}{\eta \cdot \alpha_{c}}\left(\frac{\mathrm{d} p}{\mathrm{~d} r}-\rho_{l} \cdot \omega^{2} \cdot r\right) .
$$

Integrating Eq. 14 from $r_{m}$ to $r_{c}$ leads to:

$$
p_{m}-p_{c}=\frac{\rho_{l} \cdot \omega^{2}}{2}\left(r_{m}^{2}-r_{c}^{2}\right)-\eta \cdot \alpha_{c} \cdot \frac{\mathrm{d} h}{\mathrm{~d} t}\left(r_{m}-r_{c}\right) \text {. }
$$

With:

$$
p_{m}=\eta \cdot \frac{\mathrm{d} h}{\mathrm{~d} t} \cdot R_{m}
$$

and:

$$
p_{c}=\frac{\rho_{l} \cdot \omega^{2}}{2} \cdot\left(r_{c}^{2}-r_{l}^{2}\right)
$$

integration of Eq. 14 from $t_{0}$ to $t$ results in an equation with linear dependency between the left-hand side $Y$ and the filtration time $t$.

$$
\begin{aligned}
Y & =\ln \left[\frac{r_{c}+r_{m}-h_{l}}{r_{m}-r_{c}+h_{l}} \cdot \frac{r_{m}-r_{c}+h_{l, 0}}{r_{m}+r_{c}-h_{l, 0}}\right] \\
& =\frac{\rho_{l} \cdot \omega^{2} \cdot r_{m}}{\eta \cdot\left[R_{m}+\left(r_{m}-r_{c}\right) \cdot \alpha_{c}\right]} \cdot t .
\end{aligned}
$$

This equation is valid for any filtration experiment where the membrane and filter cake position are known or can be measured. Analogous to the widely used $t / V$ - $t$ plot to determine the cake and membrane resistance in constant pressure filtration, the $Y$ - $t$ plot according to Eq. 18 allows to derive the resistances for centrifugal filtration. In this model it is assumed that the filtration process can be described one-dimensionally. A significant advantage using the LUMiSizer@ with our filtration cell is the possibility to directly measure the cake height. Thus, filter cake compression and even decompression can be taken into account and no prior knowledge of the compressibility of the material is required. To analyze just the membrane resistance with water or another liquid Eq. 18 simplifies to:

$$
Y=\ln \left[\frac{2 \cdot r_{m}-h_{l}}{h_{l}} \cdot \frac{h_{l, 0}}{2 \cdot r_{m}-h_{l, 0}}\right]=\frac{\rho_{l} \cdot \omega^{2} \cdot r_{m}}{\eta \cdot R_{m}} \cdot t
$$

\section{Material and methods}

\subsection{Lysozyme crystals}

In this study crystalline hen-egg lysozyme from Ovobest GmbH (Germany) was used. The needle shaped lysozyme 


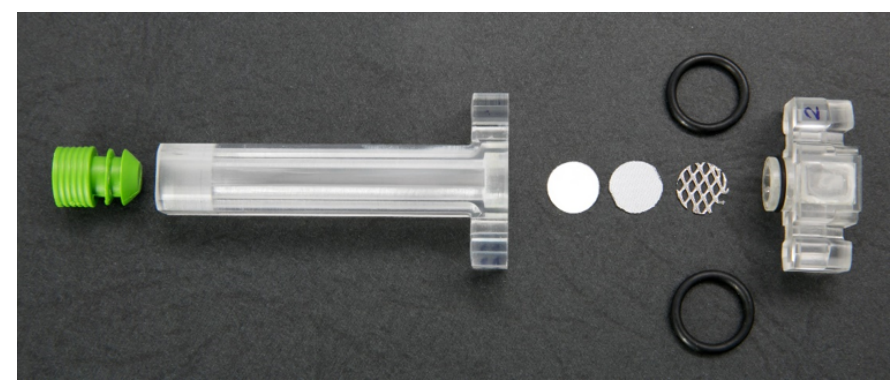

Figure 3: 3D printed filtration cell. Left: Upper part containing the suspension. Middle: Filter media and support structure. Right: Filtrate reservoir.

crystals were produced with a low temperature vacuum evaporation process described by Groß and Kind (2016); Barros Groß and Kind (2017) and kindly provided by the institute of thermal process engineering.

The isometric crystals were produced with displacement crystallization. Therefore, two stock solutions were prepared. Stock solution 1 is an acetic acid buffer at $\mathrm{pH} 4.5$, stock solution 2 contains $80 \mathrm{~g} \mathrm{~L}^{-1}$ sodium chloride additionally. For the crystallization $12.5 \mathrm{~g}$ lysozyme was dissolved in $125 \mathrm{~mL}$ stock solution 1 . With a membrane pump $125 \mathrm{~mL}$ stock solution 2 were added to the mixture at a rate of $1 \mathrm{~mL} \mathrm{~min}{ }^{-1}$. The mixture was stirred with a blade stirrer for at least $12 \mathrm{~h}$ at $300 \mathrm{rpm}$. In Table 1 the particle properties are summarized. The particle diameter shown in Table 1 was determined with static laser scattering (Sympatec Helos, Germany).

Table 1: Properties of the crystals used in this study

\begin{tabular}{lclc}
\hline Material & $\begin{array}{c}\text { Density/ } \\
\mathrm{kg} \mathrm{m}^{-3}\end{array}$ & $\begin{array}{l}\text { Particle } \\
\text { shape }\end{array}$ & $\begin{array}{c}\text { Median particle } \\
\text { diameter } / \mu \mathrm{m}\end{array}$ \\
\hline Lysozyme & 1330 & isometric & 42.5 \\
Lysozyme & 1330 & needle & 7.4 \\
\hline
\end{tabular}

\subsection{Filtration cell}

The filtration cell, shown in Fig. 3, is a 3D-print of a translucent polymer, printed at the institute of functional interfaces. It was designed to fit into the standard cuvette holders of the LUMiSizer@ centrifuges. Fig. 3 shows on the left-hand side the upper part of the cuvette, which contains the suspension and can be sealed with the standard LUM cuvette caps (left). On the right-hand side of Fig. 3 is the filtrate reservoir. In between those two parts one can put the desired filter media. If filter membranes are used, a metal support structure or support cloth must be added to prevent bending of the filter membrane. O-rings are used to fixate and seal the two parts. At the very top of the filtration cell is a small hole. At the top of the filtrate reservoir are two holes. These holes are required to release the air inside of the reservoir and thus to prevent a pressure increase in the bottom part of the filtration cell.

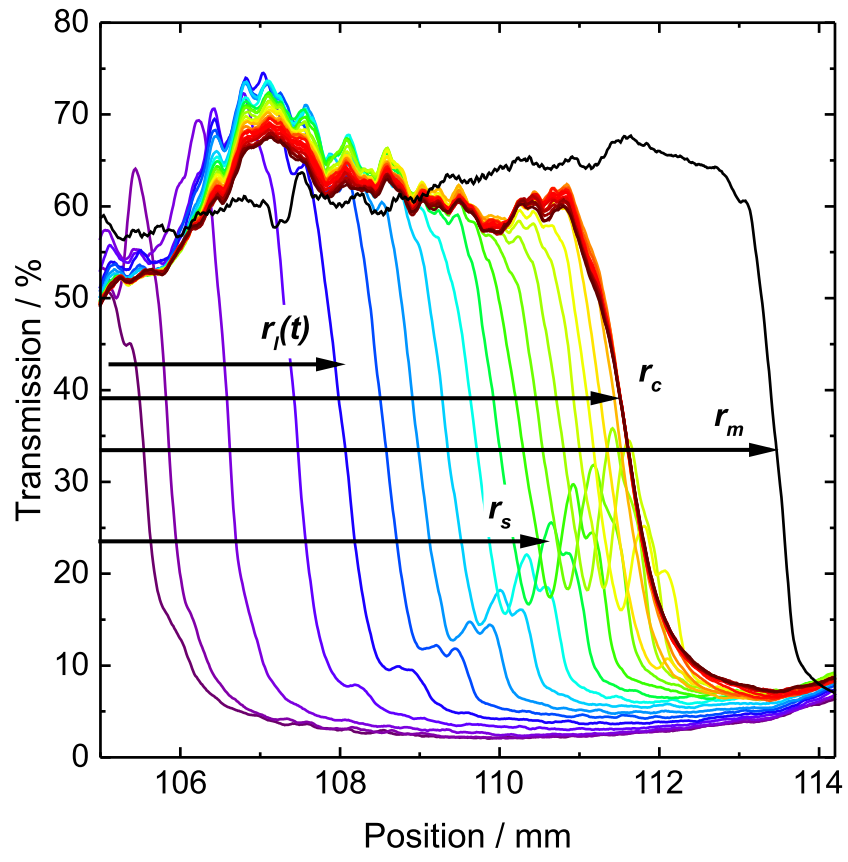

Figure 4: Example transmission profiles from a typical filtration experiment at $500 \mathrm{rpm}$. The $\mathrm{x}$-axis shows the distance from the centrifuge center, the steep parts show the liquid-gas interface. The colors represent different time-steps during the filtration process (from blue to red). The black curve shows the transmission profile of an empty filtration cell. At about 25\% transmission from position 108 to $112 \mathrm{~mm}$ a secondary peak shows the sedimentation front.

Not having those holes would lead to a different filtration behavior and to a decompression after the filtration when the centrifuge stops. The diameter of the suspension side is $8 \mathrm{~mm}$ and the cross-sectional area is $51.65 \mathrm{~mm}^{2}$. In this case the cross-sectional area also equals the filter area.

The LUMiSizer@ centrifuge used in this setup allows rotational speeds of 200 to $4000 \mathrm{rpm}$. Up to twelve samples can be processed simultaneously and the temperature can be controlled in a range from 4 to $60^{\circ} \mathrm{C}$. The wavelength of the light can be either set to near infrared light with a wavelength of $865 \mathrm{~nm}$ or to blue light with a wavelength of $470 \mathrm{~nm}$. All experiments were conducted with the $865 \mathrm{~nm}$ light source at $20^{\circ} \mathrm{C}$. The proposed setup with the filtration cell allows performing experiments with small sample volumes. Since monoclonal and therapeutic proteins are quite expensive the analysis with small sample volumes has a large cost advantage. The sample amounts used in this study ranged from 200 to $300 \mu \mathrm{L}$ per experiment.

\subsection{Analysis of the centrifugation data}

The measurement signal obtained from the centrifuge is the light transmission across the length of the cuvette. Fig. 4 shows the measurement signal from a typical filtration experiment. Only the suspension side of the filtration cell is shown as the analysis of the filtrate side has been already described by Loginov et al. (2017). Each profile has its own time stamp which is color coded in Fig. 4 from blue to red. The gas-liquid interface $\left(r_{l}\right)$ at the steep parts 
of the transmission profile can be seen clearly. As the filtration progresses, the interface moves right towards the bottom of the filtration cell. At a transmission of 20 to $25 \%$ a secondary peak forms, which shows the build-up of the sedimentation front of the particles $\left(r_{s}\right)$ in situ. This is due to the fact that in centrifugal filtration there is always a simultaneous sedimentation process leading to a clear zone of supernatant and a turbid suspension zone. Hence, it is possible to track the particle sedimentation front $\left(r_{s}\right)$. The membrane position $\left(r_{m}\right)$ can be determined by measuring an empty filtration cell (black curve). It is always required to compress the filtration cell in the centrifuge prior to the experiment at the same centrifugal speed because the manual assembly "by hand" cannot guarantee the same placement every time. Thus, small displacements will occur during the pre-compression. Also, the material of the cuvette itself will compress at different centrifugal speeds which should be taken into account when determining the membrane position.

To obtain the filtration resistance with Eq. 18 or the membrane resistance with Eq. 19 the liquid-gas interface and the sedimentation front for each profile must be determined. Also, the membrane position must be known. If the $Y$ from Eq. 18 is plotted against the time, the resulting linear curve can be interpreted analogous to the $t / V-t$ curve of a typical constant pressure filtration experiment. The slope from this curve corresponds to the right-hand side of Eq. 18 and thus allows to calculate the filter cake or membrane resistance of the filtration experiment.

\subsection{Filtration procedures}

Basically two filtration procedures are possible with this setup. One method would be to generate the filter

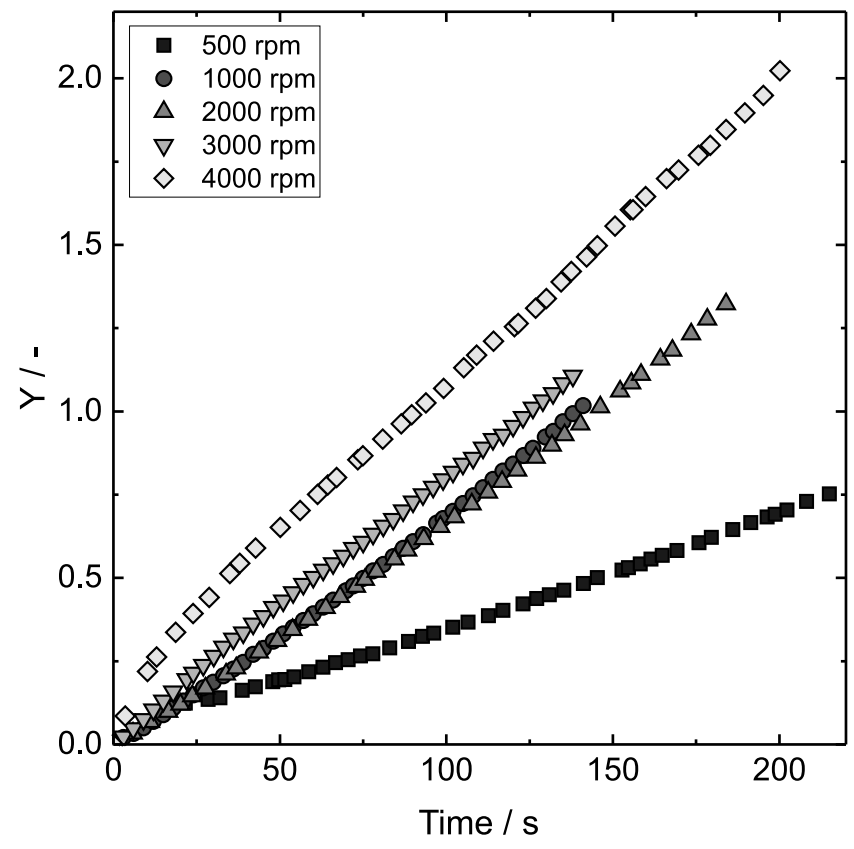

Figure 5: Exemplary $Y-t-$ curves for filter cake wash of needle shaped lysozyme crystals at various centrifugal speeds. cake and perform the filtration experiment at the same time. The other possibility is to first generate the filter cake in the centrifuge and in a second step add supernatant and wash the filter cake using the same parameters for the centrifugation. Supernatant is used to prevent the dissolution of the crystals. It has the same composition as lysozyme crystallization buffer but is particle free. For the isometric crystals the buffer is a sodium acetate buffer at $\mathrm{pH} 4.5$ with $40 \mathrm{~g} \mathrm{~L}^{-1}$ sodium chloride and $\approx 50 \mathrm{~g} \mathrm{~L}^{-1}$ dissolved lysozyme. In the case of the needle shaped crystals the buffer is a citric acid buffer at $\mathrm{pH} 4.5$ with $\approx 8 \mathrm{wt} \%$ sodium chloride and $\approx 3 \mathrm{wt} \%$ dissolved lysozyme. The exact composition of these buffers, especially for the needle shaped crystals, depends on the final composition of the suspension after crystallization. The distinction of the gas-liquid interface and the sedimentation front from the transmission profiles can be difficult. In such a case a filter cake wash is much easier to realize. When washing the filter cake there is no secondary peak indicating the sedimentation front in the transmission profiles and the filter cake position can be determined directly from each transmission profile. The maximum pressure on the filter cake and the filter membrane is at the very beginning of the filtration. This maximum pressure was calculated and used to plot the obtained results pretending that there is no influence of the pressure decrease on the filtration properties. However, this is only justified if the $Y$-t-curve shows a linear correlation with time. The filtration resistance changes with time and the pressure has to be calculated for each time step, if the filter cake decompresses or there is now linear correlation. Fig. 5 shows typical $Y-t$ curves for the washing of a filter cake consisting of needle shaped lysozyme crystals at centrifugal speeds ranging from 500 to $4000 \mathrm{~min}^{-1}$. As can be seen, the assumption of a linear correlation with time is justified. The filtration curves of isometric crystals are not shown for the sake of clarity but are also linear similar to those in Fig. 5.

\subsection{Analysis of crystal breakage}

To investigate the breakage of filtrated lysozyme crystals the filter cake was resuspended in supernatant. Microscopy images (Ortophlan, Leitz Park GmbH) with 100x magnification were taken from those crystals and analyzed with the software ImageJ 1.5 (Schneider et al., 2012) to obtain the equivalent particle diameter. The cumulative projection area sum distribution and the density distribution of the particles were calculated with MATLABß) 9.3 (The MathWorks, USA) and compared to the unstressed samples. The unstressed microscopy samples were prepared in exactly the same way as the stressed samples.

\section{Results}

\subsection{Pressure curve of the centrifugal filtration}

The filtration of isometric lysozyme crystals with the cake formation or the washing at various centrifugal speeds 

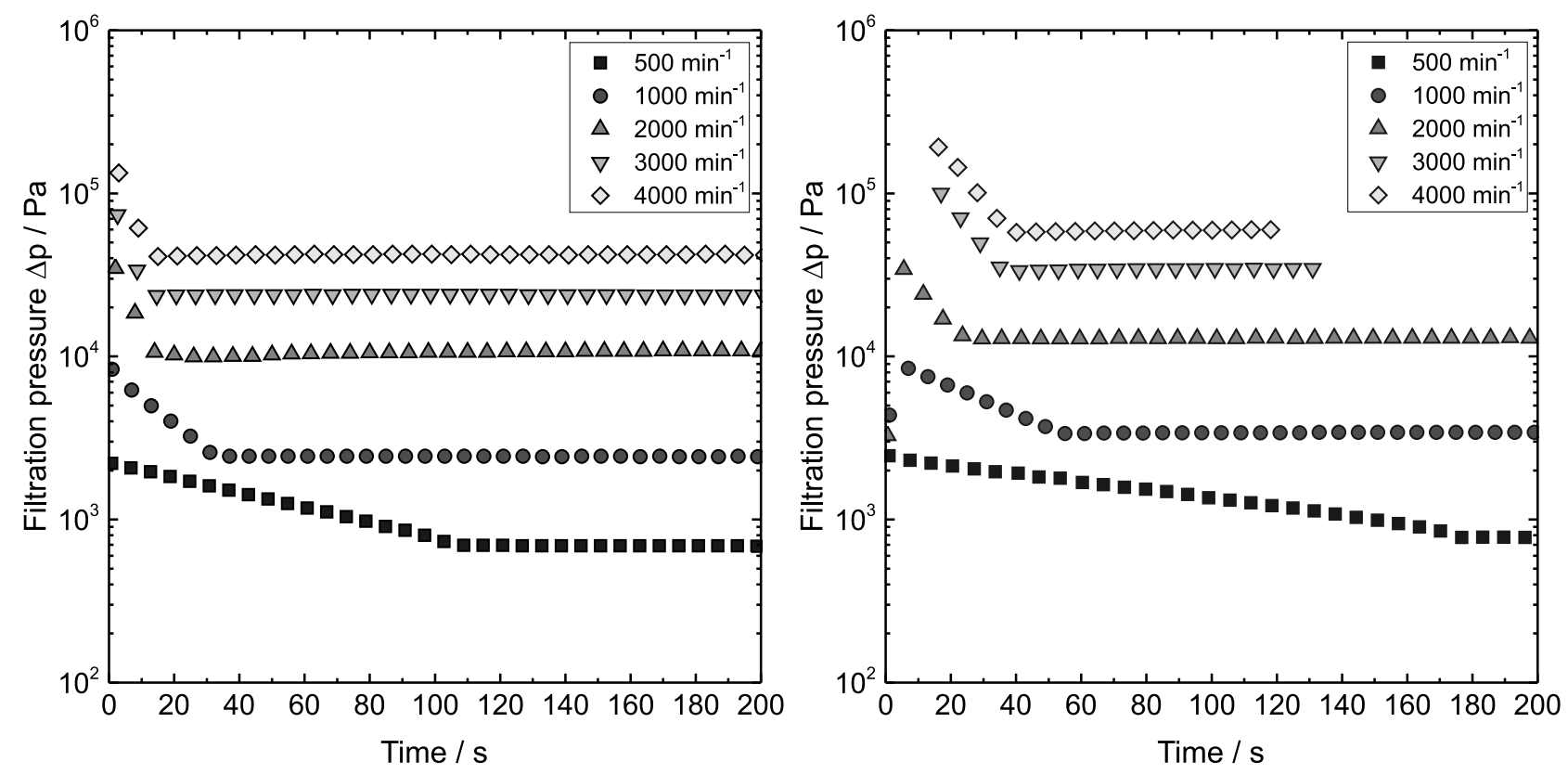

Figure 6: Left: Pressure curve of isometric lysozyme crystal filtration at different centrifugal speeds with cake formation. Right: Pressure curve of isometric lysozyme crystal cake wash at different centrifugal speeds.

leads to different pressure curves. The centrifugal speeds ranged from 500 to $4000 \mathrm{rpm}$. With the determined or calculated phase positions from the transmission profiles, the hydraulic pressure, solids pressure and pore fluid pressure are calculated using Eqs. 8-10. The sum of these pressures gives the total pressure on the filter membrane. As shown in Fig. 6, the maximum achievable pressure is about $2 \times 10^{5} \mathrm{~Pa}$. Because of small pore sizes, no dewatering of the filter cake was observed and hence the saturation of the filter cake equals one and all pores are filled with fluid. The pressure decreases with time and reaches a constant value when the whole liquid above the cake is gone. The pressure drop happens much faster at higher centrifugal speeds. This is to be expected because the angular velocity of the centrifuge has a quadratic influence on the pressure. The faster pressure drop corresponds with a shorter filtration time at higher accelerations. Hence, a filter cake wash experiment at $4000 \mathrm{rpm}$ is finished after about $40 \mathrm{~s}$. At $500 \mathrm{rpm}$ the filtration time is over $170 \mathrm{~s}$. At high accelerations (3000 and $4000 \mathrm{rpm}$ ), some data points of the cake wash experiment on the right-hand side of Fig. 6 are at the very beginning much lower. This is because the centrifuge needs a few seconds to accelerate to the desired speed. Hence, the pressure is not at the highest value at the very beginning of the filtration but when the centrifuge has reached its final speed. For the evaluation of filtration properties this has to be taken into account. That is why the experiment was only evaluated after the final speed has been reached.

\subsection{Membrane resistance}

The filter resistance of three membranes was measured by permeation with water at speeds up to $2000 \mathrm{rpm}$. At higher speeds, the permeation happens too fast and no data evaluation is possible. The filter resistance of the measured membranes are shown in Fig. 7. The membrane with a nominal pore size of $0.45 \mu \mathrm{m}$ has the lowest membrane resistance. For this membrane the measured resistance remains constant within the margin of error for all investigated speeds and has an average resistance of $5.6 \times 10^{9} \mathrm{~m}^{-1}$. The membrane with a pore size of $0.22 \mu \mathrm{m}$ has a higher resistance of $2.7 \times 10^{10}$ to $1.4 \times 10^{10} \mathrm{~m}^{-1}$. This is to be expected, because smaller pores lead to higher resistance when permeated due to the higher capillary inlet pressure. With increased centrifugal acceleration there is a small decrease in the membrane resistance. However, this decrease is still within the standard deviation. A clear difference can be observed for the membrane with the highest resistance of $2.1 \times 10^{10}$ to $3.6 \times 10^{10} \mathrm{~m}^{-1}$ and a pore size of $0.20 \mu \mathrm{m}$. The membrane resistance increases with higher centrifugal forces. This membrane is made of nylon whereas the other two membranes are made of polysulfone. Thus, it might compress at higher forces which would lead to an increase of the resistance.

Since the filter area of the membranes is only $51.65 \mathrm{~mm}^{2}$, one piece of a membrane is not enough to characterize the membrane as a whole. With a small sample number damages or uneven pore distribution of the membrane could lead to wrong conclusions. That is why the measurement has been repeated with samples taken from representative places of the membrane. Of course the value of the membrane resistance with water has only low significance when used with particulate systems. Usually the membrane resistance in such a case is about one to two decades higher. With the filtration cell it is possible to simultaneously mea- 


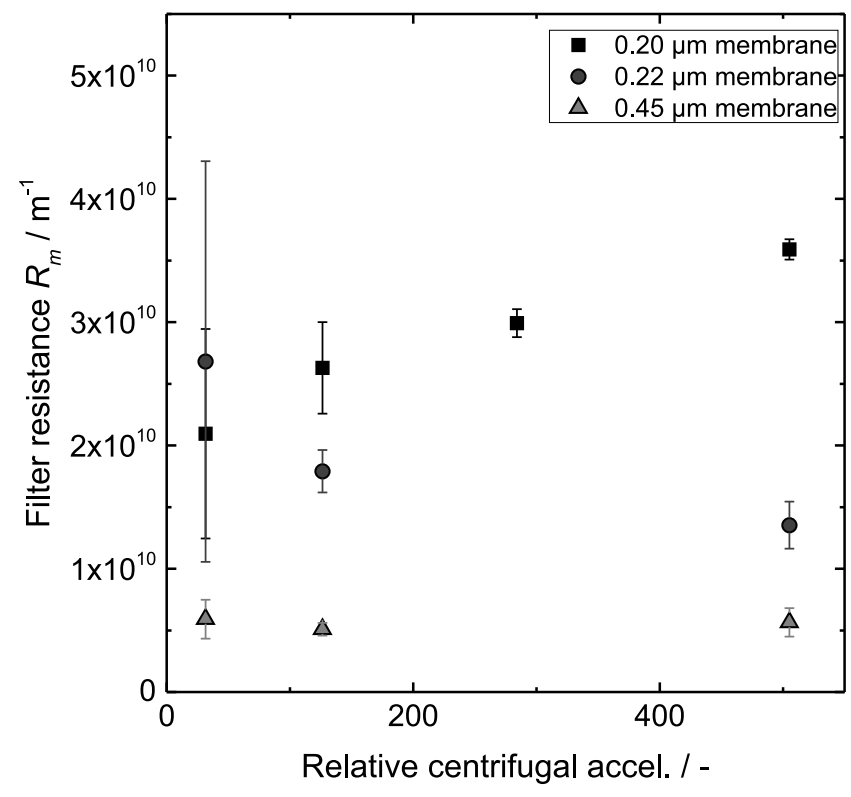

Figure 7: Membrane resistance of three different membranes when permeated with water.

sure the membrane resistance and the filter cake resistance when conducting an experiment with filter cake buildup as described in Section 3.4. In our case however, the differences in the membrane resistance are so small that they can be neglected. The main reason is that our particles are much larger than the pore size of the membranes. Also, the height specific resistance of the filter cake is several magnitudes larger than the membrane resistance so that the small error when using the water resistance value is insignificant. For all further measurements the membrane with a pore size of $0.20 \mu \mathrm{m}$ was used.

\subsection{Filter cake resistance}

The cake resistance of isometric and needle shaped lysozyme crystal filter cakes was analyzed using filter cake washing experiments as described in Section 3.4. All evaluated filter cakes were flat (visually determined) and hence the one-dimensional model from Section 2.3.2 can be applied. The properties of those two crystal systems are listed in Table 1. Obviously, a higher filtration resistance is to be expected for the smaller, needle shaped crystals. The resistances depicted in Fig. 8 increase for both investigated systems with increasing pressure. Whereas in the case of isometric lysozyme crystals the resistance at the smallest pressure of $0.02 \times 10^{5} \mathrm{~Pa}$ is $4.9 \times 10^{12} \mathrm{~m}^{-2}$ and rises to $6.8 \times 10^{13} \mathrm{~m}^{-2}$ at $1.5 \times 10^{5} \mathrm{~Pa}$, the increase is much larger in the case of needle shaped crystals. The resistance of needle shaped crystals at the very beginning is one magnitude larger than the resistance of isometric crystals. At the highest pressure the resistance is $1.3 \times 10^{16} \mathrm{~m}^{-2}$. Interestingly, the resistance of needle shaped crystals increases the most between $0.071 \times 10^{5}$ and $0.3 \times 10^{5} \mathrm{~Pa}$. The increase of $2.2 \times 10^{15} \mathrm{~m}^{-2}$ indicates a large compression of the filter cake and thus a large decrease of filter cake height. The comparison of data from the centrifugation method with a conventional experiment in the pressure filter cell (empty boxes) shows a good agreement between both methods for the isometric crystals. However, since the cake structure can be different from the pressure filter cell when using centrifugal filtration, especially with polydisperse materials, the measured data will not match exactly in most cases. Both particle systems show compressive material behavior because the resistance rises with higher pressure. The compression can be explained by restructuring of the filter cake and also by crystal breakage. Cornehl et al. (2014) demonstrated that even low pressure differences of $0.5 \times 10^{5} \mathrm{~Pa}$ can break lysozyme crystals.

\subsection{Filter cake height}

Since the filter resistance in Fig. 8 increases quite strongly for the needle shaped crystals, a decrease of the filter cake height and hence a compression of the particle bed is to be expected. In the case of incompressible material behavior one would expect the same cake height for all pressures.

For both particle systems the cake height measured with the filtration cell is shown in Fig. 9 left. The isometric crystals form a higher cake then the needle shaped crystals and the decrease of the cake height with increasing pressure is much lower than the decrease with the needle shaped crystals where the cake height decreases almost 4.5 times. Also, the settling time of the needles is much lower which leads to higher standard deviations at low centrifugal speeds and hence low pressures. At the very beginning a large height reduction of the needle shaped particle bed

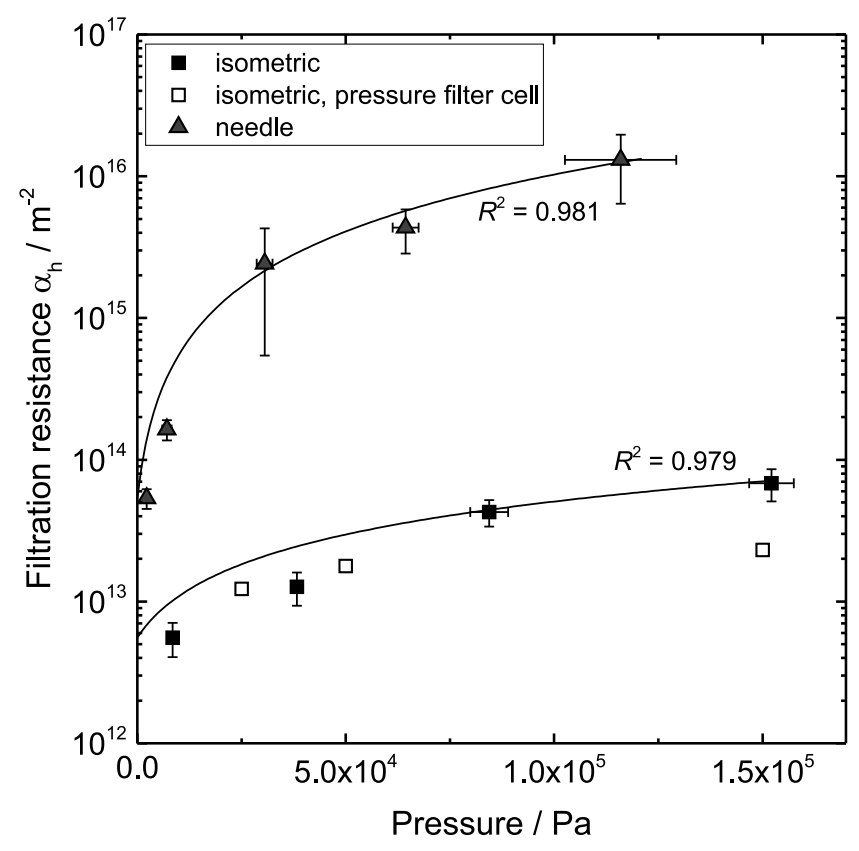

Figure 8: Height specific filtration resistance of isometric and needle shaped lysozyme crystals at different pressures in the filtration cell. The empty boxes show data obtained with a pressure filter cell as a comparison. The fit was calculated according to the empirical approach by Tiller and Kwon (1998). 

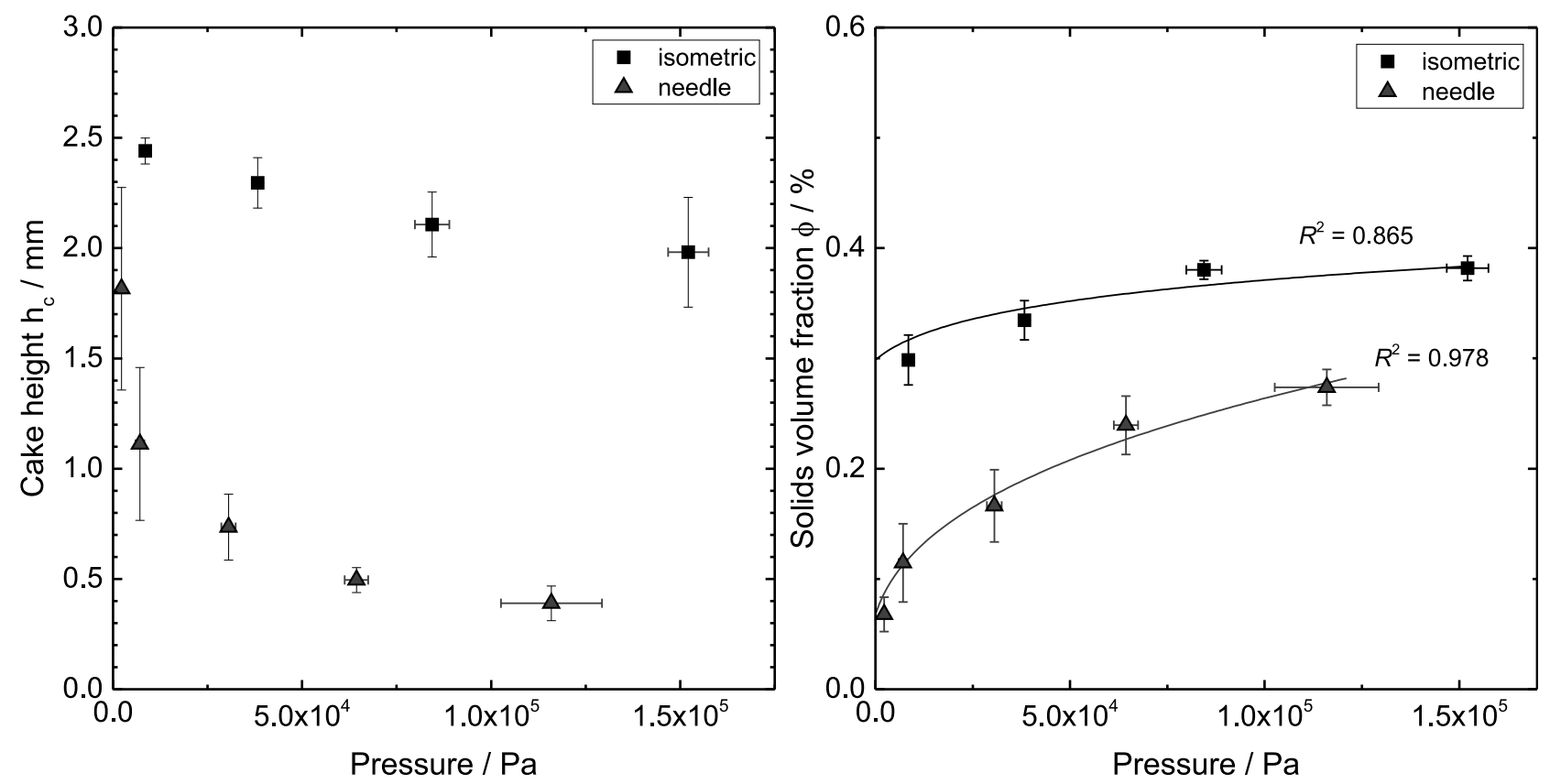

Figure 9: Left: Filter cake height for the needle shaped and isometric particle system at different pressures. Right: Solids volume fraction for isometric and needle shaped crystals at different pressures. The fit was calculated according to the empirical approach by Tiller and Kwon (1998).

can be observed, indicating a large compaction of the filter cake. At pressures larger than $0.38 \times 10^{5} \mathrm{~Pa}$ the curve flattens and the further decrease of the cake height is much smaller.

The direct measurement of the cake height is an advantage over previously published approaches (Loginov et al., 2014, 2017) because no additional experiments, assumptions or models are required. The filtration properties of a system where crystal breakage occurs can hence be measured directly.

\subsection{Measurement of the solids volume fraction}

With the knowledge of the initial volume concentration of the sample and the cake height measured with the filtration cell the solids volume fraction in the filter cake can be calculated according to Eq. 11. There are three requirements which must be met to calculate the solids volume fraction accurately. Firstly the filtration must be complete, meaning that all particles are in the filter cake. Secondly, no or a negligible amount of particles are in the filtrate and lastly the initial volume and the initial volume concentration are known. Since the used filter membrane has a pore diameter of $0.20 \mu \mathrm{m}$ and is thus much smaller than the particles the assumption that all particles are retained is justified.

The development of the solids volume fraction with increasing pressure is shown in Fig. 9 right. Due to the compaction of the filter cake the solids volume fraction increases. This effect is most evident with the needle shaped crystals because those crystals were compressed the most. In the case of the isometric crystals the increase in the solids volume fraction is approximately $10 \%$ but always higher than the solids volume fraction of the needle shaped crystals.

Although the filter cakes consisting of needle shaped crystals have much higher porosity, or a lower solids volume fraction respectively, the filtration resistance of those cakes is much higher compared to the ones consisting of isometric crystals. The needle shaped crystals are compacted much more at lower pressures than the isometric crystals. Also the total compaction factor at high pressures of 4.5 is much higher than the compaction of the isometric crystals of 1.3 . With regard to the filtration resistance: the needles have a height specific resistance of up to $1 \times 10^{16} \mathrm{~m}^{-2}$ which is two magnitudes larger than the resistance of the isometric crystal system. The filter resistances of the isometric crystals measured with the filtration cell are in good agreement to data obtained by conventional dead-end filtration in a pressure filter cell. However, if the material is polydisperse, then the cake structure can differ between the centrifugal filtration and the typical dead-end filtration. In this case the results between the two different methods might be difficult to compare.

Table 2: Fitted parameters according to Tiller and Kwon (1998) for the resistance and solids volume fraction.

\begin{tabular}{lccccc}
\hline Crystal shape & $\alpha_{0} / \mathrm{m}^{-2}$ & $\phi_{0} /-$ & $p_{0} / \mathrm{Pa}$ & $N /-$ & $\beta /-$ \\
\hline needle & $5.350 \times 10^{13}$ & 0.068 & 2244.3 & 1.377 & 0.355 \\
isometric & $5.571 \times 10^{12}$ & 0.299 & 8493.6 & 0.867 & 0.085 \\
\hline
\end{tabular}




\subsection{Compressibility}

Table 2 shows the obtained parameters from the fit of the data shown in Fig. 8 and Fig. 9 right with the approach by Tiller Eqs. 5 and 6. According to Alles and Anlauf (2003) the sum of the parameters $N$ and $\beta$ allows to characterize the compressibility of the particle system. In the case of needle shaped crystals the sum equals 1.73 and can therefore by characterized as highly compressible. The sum of exponents for the isometric crystals equals 0.95, which indicates a compressible system.

\subsection{Crystal breakage}

The size reduction of the lysozyme crystals when stressed at $4000 \mathrm{rpm}$ is shown in Fig. 10 exemplary for the isometric crystals. With increasing centrifugal speed the cumulative sum distribution moves towards smaller particles. For clarity, only the stressed samples at $4000 \mathrm{rpm}$ are shown in Fig. 10. On the top, it can be seen that the amount of larger particles decreases when the sample was stressed. Also, the amount of smaller particles increases and the whole distribution broadens, indicating a breakage of large crystals. On the bottom side the whole distribution is shifted towards smaller particle sizes when the sample has been stressed. The median particle diameter decreases from $48 \mu \mathrm{m}$ for the unstressed sample to $34 \mu \mathrm{m}$ at $4000 \mathrm{rpm}$ which equals $70 \%$ of the original particle size. The decrease in particle size is first visible at $1000 \mathrm{rpm}$ and increases until up to $3000 \mathrm{rpm}$. At higher revolutions than $3000 \mathrm{rpm}$ no further reduction of the particle size is visible. Because $3000 \mathrm{rpm}$ correspond approximately $0.9 \times 10^{5} \mathrm{~Pa}$, those results are in good agreement with the results obtained by Cornehl et al. (2014) with the pressure filter cell.

\section{Conclusion}

The 3D-printed filtration cell presented in this study allows to analyze the filtration behavior at a small scale. In combination with the LUMiSizer $\AA$ centrifuge twelve filtration experiments can be conducted at the same time. For each experiment only small amounts $(200$ to $300 \mu \mathrm{L})$ of sample solution are required. The position of the filter cake height and the position of the liquid or suspension can be measured in situ during the filtration. Due to the direct measurement of the positions all other parameters such as filtration resistance, cake height, solids volume fraction or porosity are calculated without the need for complex models or preliminary experiments.

The pressure, which can be achieved during the filtration, ranges from $0.02 \times 10^{5}$ to $2 \times 10^{5} \mathrm{~Pa}$. The pressure is dependent on the centrifugal acceleration, the filling height of the cuvette and the solids volume concentration of the sample. It decreases during the filtration meaning that the maximum pressure can only be reached for a short time at the very beginning.

It is demonstrated that important filtration properties can be successfully obtained with this filtration device using lysozyme as a model substance. The crystal shape has
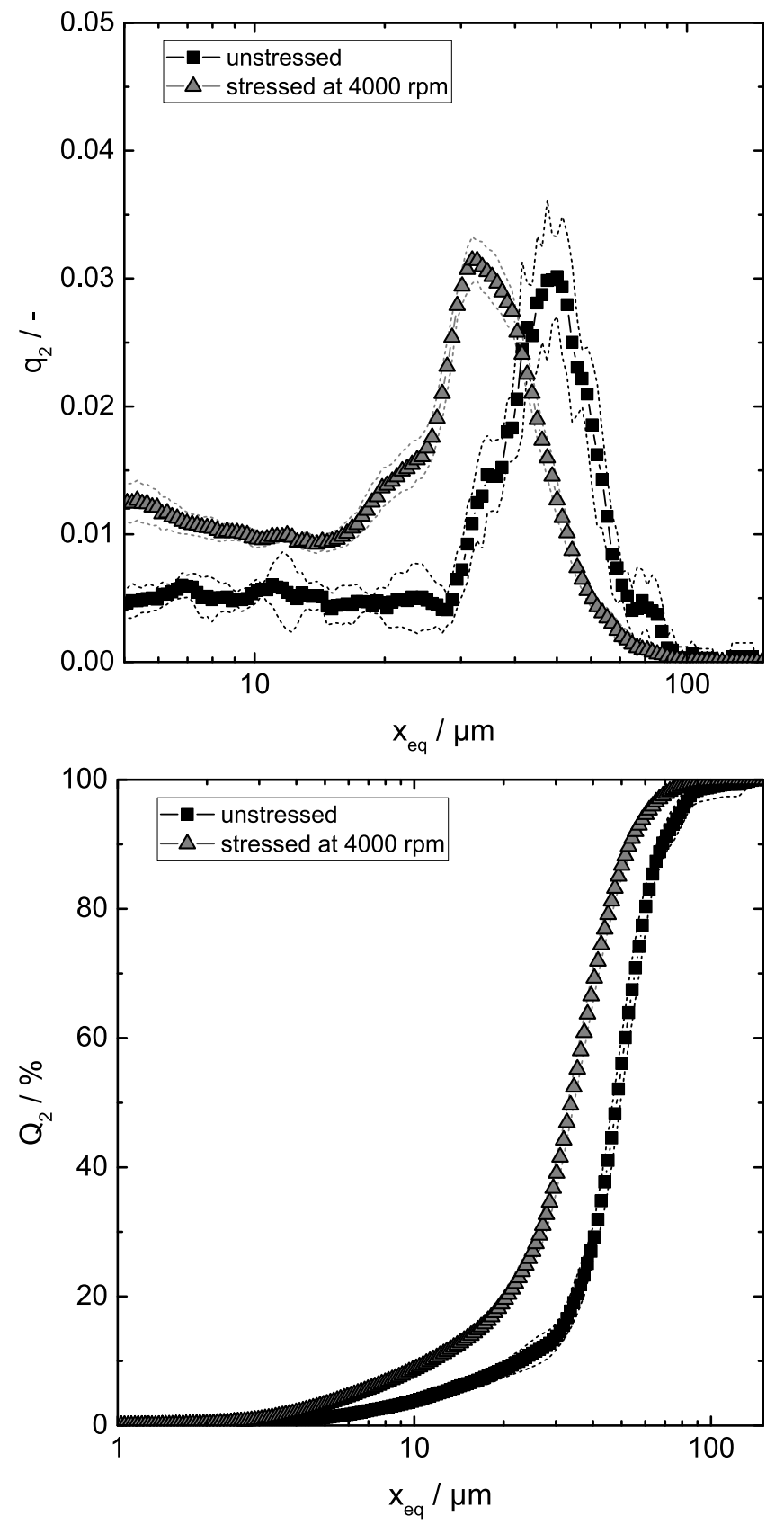

Figure 10: Top: Density distribution of unstressed and stressed isometric crystals. Bottom: Cumulative sum distribution of unstressed and stressed isometric crystals. The dashed lines show the $95 \%$ confidence region.

a large influence on the compressibility and the filtration resistance of the filter cake.

The proposed setup helps people working in the field to analyze the filterability of particles using low volume samples. Due to the high possible parallelization in the centrifuge, filtration characteristics of products can be determined faster. Furthermore, it is now possible to measure the filtration properties by measuring the liquid height above the filter cake and the cake height itself without relying on determining the filtrate mass over time. 


\section{Nomenclature}

\section{Roman symbols}

A Area

$h \quad$ Height

$K \quad$ Specific permeability

$N$ Cake resistance compressibility index

$p \quad$ Pressure

Arbitrary normalization pressure

$q \quad$ Particle density distribution

$Q$ Cumulative particle size distribu- $\%$ tion

$r \quad$ Distance from centrifuge center

$R$ Resistance

$S$ Saturation

$t$ Time

$x \quad$ Particle diameter

$\mathrm{mm}^{2}$

$\mathrm{mm}$

$\mathrm{m}^{2}$

$-$

$\mathrm{Pa}$

$\mathrm{Pa}$

$\%$

$\mathrm{mm}$

$\mathrm{m}^{-1}$

$-$

$\mathrm{S}$

$\mu \mathrm{m}$

Greek symbols

$\alpha_{0} \quad$ Height specific cake resistance at $\mathrm{m}^{-2}$ $p=p_{0}$

$\alpha_{c} \quad$ Height specific cake resistance

$\mathrm{m}^{-2}$

$\beta$ Solids volume fraction compressibility index

$\epsilon \quad$ Porosity

$\eta \quad$ Dynamic viscosity

$\rho_{s} \quad$ Solids density

$\rho_{l} \quad$ Liquid density

$\phi \quad$ Solids volume fraction

$\phi_{0} \quad$ Solids volume fraction at $p=p_{0}$

$\omega \quad$ Angular frequency

\section{Subscripts}

$0 \quad$ Initial value

c Filter cake related

$l \quad$ Liquid related

$m$ Filter medium related

$s \quad$ Suspension related

\section{Conflict of interest}

The authors declared that there is no conflict of interest.

\section{Acknowledgment}

The authors thank the German Research Foundation (DFG) for funding the project NI 414/26-1, the DiSPBiotech priority program (SPP 1934/1) and Prof. Kind and Dipl.-Ing. Michael Barros-Groß from the institute of thermal process engineering at the KIT for providing crystalline lysozyme. The helpful suggestions for improving the filtration cell and the constructive discussions with Prof. Lerche are highly appreciated.

\section{Copyright}

(C) 2018. This manuscript was accepted for publication in Chemical Engineering Science and is made available under the CC-BY-NC-ND 4.0 license: http://creativecommons.org/licenses/by-nc-nd/4.0/

The published journal article is available at:

https://doi.org/10.1016/j.ces.2018.12.019

\section{References}

Aldabaibeh, N., Jones, M.J., Myerson, A.S., Ulrich, J., 2009. The solubility of orthorhombic lysozyme crystals obtained at high ph. Cryst. Growth Des. 9, 3313-3317.

Alles, C.M., 2000. Prozeßstrategien für die Filtration mit kompressiblen Kuchen. Ph.D. thesis. Universität Karlsruhe. Karlsruhe.

Alles, C.M., Anlauf, H., 2003. Filtration mit kompressiblen Kuchen: Effiziente Konzeptefür eine anspruchsvolle Trennaufgabe. Chem. Ing. Tech. 75, 1221-1230. doi:10.1002/cite. 200303268.

Barr, J.D., White, L.R., 2006a. Centrifugal drum filtration: I. a compression rheology model of cake formation. AlChE J. 52, 545556. doi:10.1002/aic. 10678.

Barr, J.D., White, L.R., 2006b. Centrifugal drum filtration: II. a compression rheology model of cake draining. AlChE J. 52, 557564. doi:10.1002/aic. 10679

Barros Groß, M., Kind, M., 2017. Comparative study on seeded and unseeded bulk evaporative batch crystallization of tetragonal lysozyme. Cryst. Growth Des. 17, 3491-3501. doi:10.1021/acs . cgd. $7 \mathrm{~b} 00456$.

Basu, S.K., Govardhan, C.P., Jung, C.W., Margolin, A.L., 2004. Protein crystals for the delivery of biopharmaceuticals. Expert Opin. Biol. Ther. 4, 301-317. doi:10.1517/14712598.4.3.301.

Cornehl, B., Grünke, T., Nirschl, H., 2013. Mechanical stress on lysozyme crystals during dynamic cross-flow filtration. Chem. Eng. Technol. , 1665-1674doi:10.1002/ceat.201300178.

Cornehl, B., Overbeck, A., Schwab, A., Büser, J.P., Kwade, A., Nirschl, H., 2014. Breakage of lysozyme crystals due to compressive stresses during cake filtration. Chem. Eng. Sci. 111, 324-334. doi:10.1016/j.ces.2014.02.016.

Durbin, S.D., Feher, G., 1986. Crystal growth studies of lysozyme as a model for protein crystallization. J. Cryst. Growth 76, 583-592. doi:10.1016/0022-0248(86)90175-2.

Eichholz, C., Silvestre, M., Franzreb, M., Nirschl, H., 2011. Recovery of lysozyme from hen egg white by selective magnetic cake filtration. Eng. Life Sci. 11, 75-83. doi:10.1002/elsc. 201000121.

Farid, S.S., 2007. Process economics of industrial monoclonal antibody manufacture. J. Chromatogr. B 848, 8-18. doi:10.1016/j . jchromb.2006.07.037.

Groß, M., Kind, M., 2016. Bulk crystallization of proteins by lowpressure water evaporation. Chem. Eng. Technol. 39, 1483-1489. doi:10.1002/ceat.201500582.

Hekmat, D., Hebel, D., Schmid, H., Weuster-Botz, D., 2007. Crystallization of lysozyme: From vapor diffusion experiments to batch crystallization in agitated ml-scale vessels. Process Biochem. 42, 1649-1654. doi:10.1016/j.procbio.2007.10.001.

Howard, S.B., Twigg, P.J., Baird, J.K., Meehan, E.J., 1988. The solubility of hen egg-white lysozyme. J. Cryst. Growth 90, 94104. doi:10.1016/0022-0248(88)90303-X.

Jen, A., Merkle, H.P., 2001. Diamonds in the rough: Protein crystals from a formulation perspective. Pharm. Res. 18, 1483-1488.

Kayser, O. (Ed.), 2005. Pharmaceutical biotechnology: Drug discovery and clinical applications. 1.reprint ed., Wiley-VCH, Weinheim.

Koizumi, H., Tachibana, M., Kawamoto, H., Kojima †, K., 2004. Temperature dependence of microhardness of tetragonal hen-eggwhite lysozyme single crystals. Philos. Mag. 84, 2961-2968. doi:10.1080/14786430410001716791. 
Lerche, D., Sobisch, T., 2007. Consolidation of concentrated dispersions of nano- and microparticles determined by analytical centrifugation. Powder Technol. 174, 46-49. doi:10.1016/j.powtec. 2006.10.020.

Liu, Y., Wang, X., Ching, C.B., 2010. Toward further understanding of lysozyme crystallization: Phase diagram, protein-protein interaction, nucleation kinetics, and growth kinetics. Cryst. Growth Des. 10, 548-558. doi:10.1021/cg900919w.

Liu, Y.M., Li, H.S., Wu, Z.Q., Chen, R.Q., Lu, Q.Q., Guo, Y.Z., Zhang, C.Y., Yin, D.C., 2016. Sensitivity of lysozyme crystallization to temperature variation. Cryst. Eng. Comm. 18, 1609-1617. doi:10.1039/C6CE00060F.

Loginov, M., Lebovka, N., Vorobiev, E., 2014. Multistage centrifugation method for determination of filtration and consolidation properties of mineral and biological suspensions using the analytical photocentrifuge. Chem. Eng. Sci. 107, 277-289. doi:10.1010/j.ces.2013.12.011.

Loginov, M., Samper, F., Gésan-Guiziou, G., Sobisch, T., Lerche, D., Vorobiev, E., 2017. Centrifugal ultrafiltration for determination of filter cake properties of colloids. J. Membr. Sci. 536, 59-75. doi:10.1016/j.memsci.2017.04.064.

Morozov, V.N., Morozova, T.Y., 1981. Viscoelastic properties of protein crystals: Triclinic crystals of hen egg white lysozyme in different conditions. Biopolymers 20, 451-467. doi:10.1002/bip. 1981.360200304.

Rushton, A., Ward, A.S., Holdich, R.G., 2000. Solid-liquid filtration and separation technology. 2., compl. rev. ed. ed., VCH, Weinheim.

Sambuichi, M., Nakakura, H., Osasa, K., Tiller, F.M., 1987. Theory of batchwise centrifugal filtration. AlChE J. 33, 109-120. doi:10. 1002/aic.690330113.

Schmidt, S., Havekost, D., Kaiser, K., Kauling, J., Henzler, H.J., 2005. Crystallization for the downstream processing of proteins. Eng. Life Sci. 5, 273-276. doi:10.1002/elsc. 200500116.

Schneider, C.A., Rasband, W.S., Eliceiri, K.W., 2012. Nih image to imagej: 25 years of image analysis. Nat. Methods 9, 671-675.

Tait, S., White, E.T., Litster, J.D., 2008. Mechanical characterization of protein crystals. Part. Part. Syst. Charact. 25, 266-276. doi:10.1002/ppsc. 200701112.

Tiller, F.M., Hsyung, N.B., 1993. Unifying the theory of thickening, filtration, and centrifugation. Water Sci. Technol. 28, 1-9.

Tiller, F.M., Kwon, J.H., 1998. Role of porosity in filtration: Xiii. behavior of highly compactible cakes. AlChE J. 44, 2159-2167.

Walsh, G., 2002. Proteins: Biochemistry and biotechnology. Wiley, Chichester.

Zamiri, A., De, S., 2010. Modeling the mechanical response of tetragonal lysozyme crystals. Langmuir 26, 4251-4257. doi:10.1021/ 1 a9033222. 